\title{
Classical Scattering Theory in One Dimension
}

\author{
Andrea Hubacher
}

ETH, Zürich, Switzerland

\begin{abstract}
We discuss various scattering properties of the classical system of $n$ repelling particles on the real line. In the integrable case, i.e. if the asymptotic velocities are preserved under the scattering map, the asymptotic phases behave as if the particles collided pairwise.
\end{abstract}

\section{Introduction and Results}

We consider $n$ mass points on the real line interacting through the Hamiltonian

$$
H(x, y)=\sum_{k=1}^{n} \frac{y_{k}^{2}}{2}+\sum_{1 \leqq j<k \leqq n} V\left(x_{k}-x_{j}\right) .
$$

The equations of motion are

$$
\dot{x}_{k}=y_{k} ; \quad \dot{y}_{k}=-\sum_{j<k} V^{\prime}\left(x_{k}-x_{j}\right)+\sum_{j>k} V^{\prime}\left(x_{j}-x_{k}\right) .
$$

We concentrate in this paper on the question of the asymptotic behaviour of the solutions of (1.2) as time goes to infinity. We first examine conditions under which the scattering map exists and is symplectic (Theorems 1 to 3 and Example 1). Our main result concerns integrable systems, i.e. systems for which the scattering map preserves the asymptotic velocities up to permutation. We find that in integrable systems the asymptotic phases behave as if the particles were colliding pairwise (Theorem 4).

It is well known [18] that for repulsive potentials asymptotic velocities $b_{k}=\lim _{t \rightarrow \infty} \dot{x}_{k}(t)$ exist for all $k=1, \ldots, n$. One can ask a more subtle question: Are asymptotic velocities pairwise distinct, i.e. $b_{j} \neq b_{k}$ for all $j \neq k$ ? If they are, then the particles fly infinitely far apart from each other as time goes to infinity. It is already known [14] that distinctness of asymptotic velocities holds under decay properties on $V^{\prime}$, such as $V^{\prime}(x)=O\left(|x|^{-2-\alpha}\right)$ as $|x| \rightarrow \infty$ for some $\alpha>0$. We find here that for a Hamiltonian (1.1) one can give necessary and sufficient conditions 


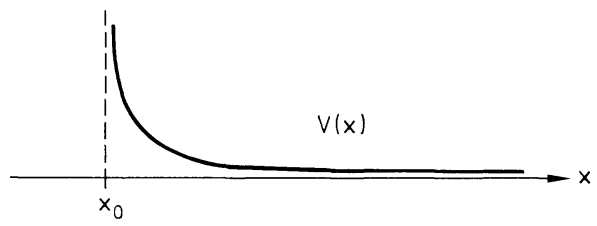

Fig. 1

that do not involve decay properties of $V^{\prime}$ for the existence of distinct asymptotic velocities.

Theorem 1. Let $V$ be $C^{1}$ on an interval $\left(x_{0}, \infty\right)$ unbounded to the right, where $x_{0}$ is some fixed real number or $-\infty$. Then up to normalization, the conditions

(A1) $V^{\prime}<0$,

(A2) $V>0$ (which means that $V$ is bounded from below),

(A3) $V(x) \rightarrow \infty$ as $x \rightarrow x_{0}^{+}$

are necessary and sufficient for all solutions of (1.2) to exist globally and to have asymptotic velocities which are pairwise distinct and have the ordering

$$
\dot{x}_{1}(\infty)<\dot{x}_{2}(\infty)<\ldots<\dot{x}_{n}(\infty) \text {. }
$$

Figure 1 shows the shape of a potential $V$ satisfying (A1)-(A3).

Next we ask for existence of the asymptotic phases $a_{k}=\lim \left(x_{k}(t)-t \dot{x}_{k}(\infty)\right)$ for $k=1, \ldots, n$. This question has a nice answer due to Galperin [6]: Under the assumption that the potential is repulsive and that the asymptotic velocities are pairwise distinct, the condition

$$
\int^{\infty}\left|x V^{\prime}(x)\right| d x<\infty
$$

is necessary and sufficient for existence of the asymptotic phases. We warn the reader that for this separation of the conditions (A 1)-(A4) (i.e. only (A1)-(A3) being needed for the asymptotic velocities and only (A4) being needed for the asymptotic phases), the special form (1.1) of the Hamiltonian is crucial. The sufficiency statement of Theorem 1 is not valid for a Hamiltonian

$$
H(x, y)=\sum_{k=1}^{n} \frac{y_{k}^{2}}{2 m_{k}}+\sum_{k<j} V_{k j}\left(x_{k}-x_{j}\right)
$$

with each $V_{k j}$ satisfying (A1)-(A3). The following counterexample demonstrates this.

Example 1. Consider the 3 particle system with Hamiltonian

$$
H(x, y)=\sum_{k=1}^{3} \frac{y_{k}^{2}}{2}+e^{x_{1}-x_{2}}+e^{x_{1}-x_{3}}+2\left|x_{1}-x_{2}\right|^{-1 / 2} .
$$

This system has for any $b=\left(b_{1}, b_{2}, b_{3}\right)$ with $b_{1}=b_{2}<b_{3}$ a solution $x(t)=\left(x_{1}(t)\right.$, $\left.x_{2}(t), x_{3}(t)\right)$ with $\dot{x}(\infty)=b$. So the asymptotic velocities are not distinct.

For a Hamiltonian (1.4) we therefore need decay properties on $V_{k j}^{\prime}$ to insure distinct asymptotic velocities. We find that it is sufficient to add the very weak 
condition (A 4) of Galperin in order to obtain distinct asymptotic velocities; hence (A1)-(A4) are for a Hamiltonian (1.4) still the best conditions for both distinctness of asymptotic velocities and existence of asymptotic phases:

Theorem 2. If each $V_{k j}$ in (1.4) satisfies (A1)-(A4), then the asymptotic velocities are pairwise (and therefore by Galperin [6] the asymptotic phases also exist).

Let us return to system (1.1). We assume now that $V$ is $C^{2}$, satisfies (A1)--(A 3) and for some $\alpha>0$ has the decay properties

$$
\left|V^{\prime}(x)\right| \leqq M / x^{2+\alpha}, \quad\left|V^{\prime \prime}(x)\right| \leqq M / x^{3+\alpha} \quad \text { for all } \quad x \geqq R .
$$

Since $V$ is $C^{2}$, the solutions of (1.2) are unique. Therefore, by Theorem 1 and Galperin [6] we can define the wave operator $\sigma^{+}:(x, y) \rightarrow(a, b)$ which associates to the point $(x, y)$ of the phase space the asymptotic phases $a$ and the asymptotic velocities $b$ of the orbit starting at $(x, y)$. By Theorem $1, \sigma^{+}$maps the phase space into the set

$$
A^{+}=\left\{(a, b) \in \mathbb{R}^{n} \times \mathbb{R}^{n} \mid b=\left(b_{1}, \ldots, b_{n}\right) \text { and } b_{1}<b_{2}<\ldots<b_{n}\right\} .
$$

By Simon [16], any $n$ particle system (not necessarily repulsive) with interaction potential decaying like $V^{\prime}(x)=O\left(|x|^{-2-\alpha}\right), V^{\prime \prime}(x)=O\left(|x|^{-3-\alpha}\right)$ as $|x| \rightarrow \infty$ contains a nonempty open subsystem on which wave operators exist, and are homeomorphismus to appropriate sets of asymptotic data. By Herbst [8] these wave operators are even symplectic. Applying the results of Simon [16] and Herbst [8] to our system (1.1) gives that $\sigma^{+}$is a symplectic diffeomorphism from a nonempty open subset of the phase space onto $A^{+}$. By Theorem 1, this subset equals the whole phase space. Hence the following theorem holds:

Theorem 3. If $V$ satisfies (A1)-(A 3) and (B), then $\sigma^{+}$is a symplectic diffeomorphism from the phase space onto $A^{+}$.

From Theorems 1 and 3 we obtain the other wave operator $\sigma^{-}$by time inversion. $\sigma^{-}$maps the phase space symplectically and diffeomorphically onto the set

$$
A^{-}=\left\{(a, b) \in \mathbb{R}^{n} \times \mathbb{R}^{n} \mid b=\left(b_{1}, b_{2}, \ldots, b_{n}\right) \text { and } b_{1}>b_{2}>\ldots>b_{n}\right\} .
$$

Thus the scattering map $\sigma=\sigma^{+}\left(\sigma^{-}\right)^{-1} . A^{-} \rightarrow A^{+}$exists and is symplectic.

The symplecticity of the wave operator $\sigma^{+}$(respectively $\sigma^{-}$) implies that the asymptotic velocities at plus infinity (respectively at minus infinity) are $n$ independent integrals of the motion which are in involution. Integrability, in the sense of existence of $n$ independent integrals in involution for our system is however not surprising since the flow is globally parallel. Any system with globally parallel flow is integrable in this sense. But the systems we now consider, which we will call integrable, have strong additional algebraic properties which lead to the preservation of the asymptotic velocities in the transition from minus to plus infinity. Let us give a precise definition:

Definition. We call a system (1.1) integrable if the potential $V$ satisfies (A1)-(A3) and (B) and if for each $n \geqq 2$ the scattering map $\sigma: A^{-} \rightarrow A^{+}$, $\left(a^{-}, b^{-}\right) \rightarrow\left(a^{+}, b^{+}\right)=\sigma\left(a^{-}, b^{-}\right)$acts on the asymptotic velocities according to

$$
b_{n-k+1}^{+}=b_{k}^{-} \quad \text { for } k=1, \ldots, n \text {. }
$$


Examples are the Calogero potential $V(x)=1 / x^{2}[14]$, the Toda potential $V(x)=e^{-x}$ [13], and the potential $V(x)=1 / \sinh ^{2}(x)$ [2]. (To be precise: The Hamiltonian of the integrable system with Toda potential $e^{-x}$ is not (1.1), but rather the nearest neighbor Hamiltonian

$$
H(x, y)=\sum_{k=1}^{n} \frac{y_{k}^{2}}{2}+\sum_{k=1}^{n-1} V\left(x_{k+1}-x_{k}\right) .
$$

But all results of this paper are obviously valid for a nearest neighbor Hamiltonian.)

We show that integrability (1.8) and the decay condition (B) are sufficient to calculate the whole scattering map explicitly. Symplecticity of the scattering map is the same as

$$
\sum_{k=1}^{n} d a_{k}^{-} \wedge d b_{k}^{-}=\sum_{k=1}^{n} d a_{k}^{+} \wedge d b_{k}^{+} .
$$

This combined with (1.8) gives that

$$
d\left(\sum_{k=1}^{n}\left(a_{n-k+1}^{+}-a_{k}^{-}\right) d b_{k}^{-}\right)=0 .
$$

Hence, since the domain $A^{-}$of $\sigma$ is simply connected one finds

$$
a_{n-k+1}^{+}-a_{k}^{-}=\partial G_{n} / \partial b_{k}^{-}=\delta_{k},
$$

where $G_{n}$ is a function of $b^{-}=\left(b_{1}^{-}, b_{2}^{-}, \ldots, b_{n}^{-}\right)$only. The phase shifts $\delta_{k}=\partial G_{n} / \partial b_{k}^{-}$are then independent of the phases $a_{1}^{-}, \ldots, a_{n}^{-}$. Because the phase shifts are translation invariant (i.e. independent of the motion of the center of mass), $G_{n}$ is a function of the differences $b_{j}^{-}-b_{k}^{-}$only. But we can even say more:

Theorem 4. Let the potential $V$ satisfy (A1)-(A3) and (B). Assume that the $n$ particle system is integrable, i.e. satisfies (1.8) for each $n \geqq 2$. The symplecticity of the scattering map means that (1.9) is valid. Moreover

$$
G_{n}\left(b^{-}\right)=\sum_{j<k} G_{2}\left(b_{j}^{-}-b_{k}^{-}\right) .
$$

Therefore the phase shifts have the form

$$
\delta_{k}=-\sum_{j<k} \delta\left(b_{j}^{-}-b_{k}^{-}\right)+\sum_{j>k} \delta\left(b_{k}^{-}-b_{j}^{-}\right),
$$

where $\delta=G_{2}^{\prime}$ is the two particle phase shift.

This means that the phase shifts are entirely determined by the pairwise interaction. Thus for these special systems the determination of the scattering map is reduced to the determination of $G_{2}\left(b^{-}\right)$, or equivalently to the computation of the phase shifts of a two particle system which can be done by explicit integration. By Moser [15] the two particle phase shifts are given by the Abelian integral equation

$$
\delta(\beta)=-V^{-1}(E)+\int_{V-1(E)}^{\infty}\left(\frac{\sqrt{E}}{\sqrt{E-V(x)}}-1\right) d x, \quad \text { where } \quad E=\beta^{2} / 4
$$




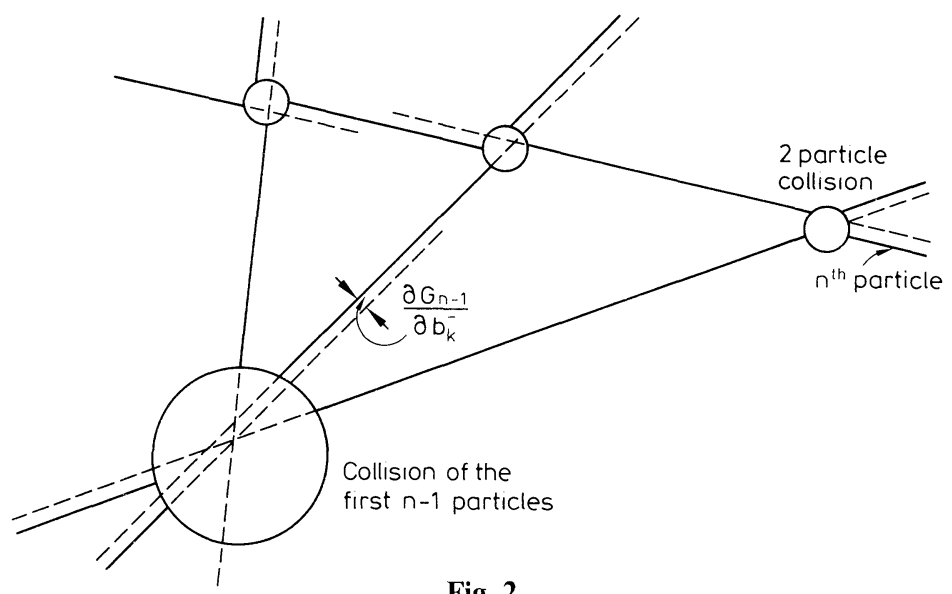

Fig. 2

For the Calogero-potential $1 / x^{2}$ one finds $\delta=0$ [1]; for the Toda-potential $e^{-x}$ one finds $\delta(\beta)=\log \beta^{2}$ [13]. The proof of Theorem 5 is by induction over the number of particles. For the induction procedure we are motivated by the following geometrical picture by Moser [15] (see also Kulish [12]): We assume (1.10) respectively (1.11) to be valid for $n-1$ particles. To make the induction step from $n-1$ to $n$, we choose in the $n$ particle system the asymptotic phase $a_{n}^{-}$of the $n^{\text {th }}$ particle so large that when the $n^{\text {th }}$ particle approaches the particles 1 to $n-1$, these first $n-1$ particles have already undergone their interaction, are moving asymptotically free and are so far apart from each other, that the " $n^{\text {th }}$ particle collides pairwise with each of them". See Fig. 2.

\section{Some Related Literature}

Hunziker [9] proved that for potentials with compact support (not necessarily repulsive) almost all orbits with unbounded distance between the particles collide finitely often and that these systems are asymptotic complete. Galperin [4] (see also Sinai [17] and Galperin [5]) proved that finitely many mass points on the real line interacting by perfectly elastic collisions collide finitely often. Theorems 1 and 2 can be interpreted in the following way: Particles interacting by repulsive potentials which have an infinite support also "collide" finitely many times.

Moser [15] proved that the Calogero-potential $1 / x^{2}$ is the only integrable system with phase shifts identically zero. Khimchenko and Sinai [11] proved that a potential with compact support never gives rise to an integrable system and that the Calogero potential $1 / x^{2}$ is the only potential in the class of potentials decaying as $V(x) \sim x^{-\alpha}, V^{\prime}(x) \sim x^{-1-\alpha}, V^{\prime \prime}(x) \sim x^{-2-\alpha}$ for some $\alpha \geqq 2$ generating an integrable system.

The phenomenon described by Theorem 4 is reminiscent of the behaviour of other integrable systems. For $N$-soliton solutions of the Korteweg de Vries equation $u_{t}+u \cdot u_{x}+u_{x x x}=0$, the total phase shift is just the sum of the shifts that would occur in isolated pairwise interaction with every other soliton (Gardner, 
Greene, Kruskal, Miura [7], Theorem 3.7, p. 122). There exist connections between the $\mathrm{KdV}$ equation and one dimensional integrable $n$ particle systems. For rational solutions $u(x, t)=\Sigma 2 /\left(x-a_{i}(t)\right)^{2}$ of $\mathrm{KdV}$, the time evolution of the poles $a_{i}(t)$ is given by the Hamiltonian flow generated by a certain integral of the $n$ particle system with Calogero potential $1 / x^{2}$ (see [1 and 3]).

\section{Proof of Theorems 1 and 2}

For the proof of Theorems 1 and 2 we need the following lemma.

Lemma. Let $f(r)>0$ be continuous for $r \geqq 0$ and $\int_{0}^{\infty} f(r) d r<\infty$. Let $r(t) \geqq 0$ and $r_{0}(t) \geqq 0$ be continuously differentiable for $t \geqq 0$ and $\dot{r}_{0}(t) \rightarrow 0, \dot{r}(t) \rightarrow b>0$ as $t \rightarrow \infty$. Furthermore let $\int_{0}^{\infty} f\left(r_{0}(t)\right) d t<\infty$. Then the following holds:

(i) $\int_{0}^{\infty} f(r(t)) d t<\infty$,

(ii) $r_{0}(t) \rightarrow \infty$ as $t \rightarrow \infty$.

(iii) For any $\varepsilon>0$ there exists $T \geqq 0$ such that $\int_{t}^{\infty} f(r(s)) d s \leqq \varepsilon \int_{t}^{\infty} f\left(r_{0}(s)\right) d s$ for all $t \geqq T$.

Proof of the lemma. (i) and (ii) are obvious. Proof of (iii): For any $\varepsilon>0$ there exists $T \geqq 0$ such that $r_{0}(s) \leqq r(s),\left|\dot{r}_{0}(s)\right| \leqq \varepsilon b / 2$, and $\dot{r}(s)>b / 2$ for all $s \geqq T$. Then we have for all $t \geqq T$ that

$$
\begin{aligned}
\varepsilon b / 2 & \int_{t}^{\infty} f\left(r_{0}(s)\right) d s \geqq\left|\int_{t}^{\infty} f\left(r_{0}(s)\right) \dot{r}_{0}(s) d s\right|=\int_{r_{0}(t)}^{\infty} f(r) d r \geqq \int_{r(t)}^{\infty} f(r) d r \\
= & \int_{t}^{\infty} f(r(s)) \dot{r}(s) d s \geqq b / 2 \int_{t}^{\infty} f(r(s)) d s .
\end{aligned}
$$

Proof of Theorem 1. Necessity of (A1)-(A 3): $V^{\prime}$ has no zeros; otherwise the system would have equilibrium points, which are not solutions with distinct asymptotic velocities. Therefore either $V^{\prime}>0$ or $V^{\prime}<0$. The fixed ordering (1.3) of the asymptotic velocities forces $V^{\prime}<0$, otherwise one could choose initial conditions, such that this ordering is violated. $V$ has to be bounded from below. Otherwise $V^{\prime}<0$ implies $V(x) \rightarrow-\infty$ as $x \rightarrow \infty$, and any solution $x_{1}(t)$, $x_{2}(t), \ldots, x_{n}(t)$ of (1.2) with the property $x_{k}(t)-x_{j}(t) \rightarrow \infty$ as $t \rightarrow \infty$ for $k>j$, would have unbounded kinetic energy, i.e. $\Sigma x_{k}^{2}(t) \rightarrow \infty$ as $t \rightarrow \infty$, contradicting the existence of asymptotic velocities. Without loss of generality we can assume $V>0$. The infinite repulsivity (A 3 ) is in the case $x_{0}>-\infty$ necessary for global existence of the solutions; in the case $x_{0}=-\infty$ it is necessary for the distinctness of asymptotic velocities. To make this point clearer, look at Fig. 3 which shows the shape of a potential with $x_{0}=-\infty$ and $\lim _{x \rightarrow-\infty} V(x)<\infty$. For $n=2$, one easily sees that there exists an orbit with equal asymptotic velocities, namely the orbit with relative energy $\lim _{x \rightarrow-\infty} V(x)$. The analogue phenomenon occurs for $n>2$.

Sufficiency of (A1)-(A3): (A1)-(A3) are sufficient for global existence of the solutions of (1.2). (We do not need uniqueness of the solutions.) 


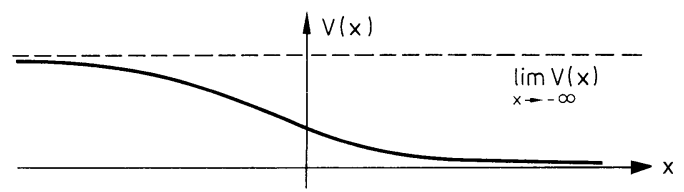

Fig. 3. Shape of $V(x)$ if $x_{0}=-\infty$ and $\lim _{x \rightarrow-\infty} V(x)<\infty$. The two particle orbit with relative energy $\lim _{x \rightarrow-\infty} V(x)$ has not distinct asymptotic velocities

Let $x(t)=\left(x_{1}(t), \ldots, x_{n}(t)\right), y(t)=\left(y_{1}(t), \ldots, y_{n}(t)\right)$ be an orbit of (1.2). $V^{\prime}<0$ implies that $\frac{d}{d t}\left(\sum_{k=1}^{j} y_{k}\right)=\sum_{\substack{q \leqq j \\ r>j}} V^{\prime}\left(x_{r}-x_{q}\right) \leqq 0$ for all $j$. Therefore $\sum_{k=1}^{j} y_{k}(t)$ is monotonously decreasing; because of energy conservation and $V>0$ it stays

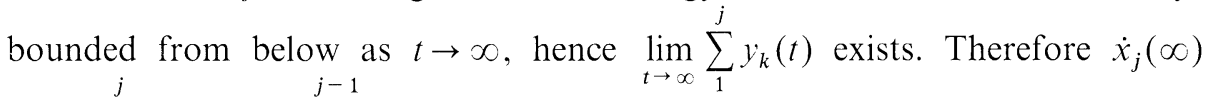
$=\lim _{t \rightarrow \infty} \sum_{1}^{j} y_{k}(t)-\lim _{t \rightarrow \infty} \sum_{1}^{j-1} y_{k}(t)$ exists for all $j$.

Energy conservation and $V(x) \rightarrow \infty$ as $x \rightarrow x_{0}^{+}$imply for all $j<k$ boundedness of $x_{k}(t)-x_{j}(t)$ from below as $t \rightarrow \infty$, from which there follows the ordering

$$
\dot{x}_{1}(\infty) \leqq \dot{x}_{2}(\infty) \leqq \ldots \leqq \dot{x}_{n}(\infty) \text {. }
$$

Equations (1.2) imply that $\sum_{\substack{q \leqq j \\ r>j}} \int_{\substack{j \\ j}}^{t} V^{\prime}\left(x_{r}(s)-x_{q}(s)\right) d s=\sum_{k=1}^{j} y_{k}(t)-\sum_{k=1}^{j} y_{k}(0)$ for all $t \geqq 0$ and for all $j=1, \ldots, n$; from which we conclude by (A1) and the existence of the asymptotic velocities that $\int_{0}^{\infty}\left|V^{\prime}\left(x_{r}(s)-x_{q}(s)\right)\right| d s<\infty$ for all $q<r$. The existence of these integrals, the ordering (2.1), and the strict repulsivity (A1) lead by statement (ii) of the lemma to $x_{r}(t)-x_{q}(t) \rightarrow \infty$ as $t \rightarrow \infty$, for all $q<r$. Now we prove that the asymptotic velocities are distinct: Assume that $\dot{x}_{j}(\infty)=\dot{x}_{k}(\infty)$ for some $j<k$. It is no restriction to assume that

$$
\dot{x}_{j-1}(\infty)<\dot{x}_{j}(\infty) \quad(\text { if } j>1) \quad \text { and } \quad \dot{x}_{k}(\infty)<\dot{x}_{k+1}(\infty) \quad(\text { if } k<n) .
$$

Observe that from now on we keep $j$ and $k$ fixed. $\dot{x}_{k}(\infty)-\dot{x}_{j}(\infty)=0$ implies that $\dot{x}_{k}(t)-\dot{x}_{j}(t)=-\int_{t}^{\infty} \ddot{x}_{k}(s)-\ddot{x}_{j}(s) d s$, from which by inserting Eqs. (1.2) and by using (A1), (2.2) and the statement (iii) of the lemma we obtain that for $\varepsilon>0$ and large $t$,

$$
\begin{aligned}
\dot{x}_{k}(t)-\dot{x}_{j}(t) & <2 \int_{t}^{\infty} V^{\prime}\left(x_{k}(s)-x_{j}(s)\right) d s-\sum_{r>k} \int_{t}^{\infty} V^{\prime}\left(x_{r}(s)-x_{k}(s)\right) d s \\
& -\sum_{r<j} \int_{t}^{\infty} V^{\prime}\left(x_{j}(s)-x_{r}(s)\right) d s \\
& \leqq(-2+\varepsilon(n-k)+\varepsilon(j-1)) \int_{t}^{\infty}\left|V^{\prime}\left(x_{k}(s)-x_{j}(s)\right)\right| d s .
\end{aligned}
$$


For $\varepsilon$ small and $t$ large, the above inequality implies that $\dot{x}_{k}(t)-\dot{x}_{j}(t)<0$, which implies that $x_{k}(t)-x_{j}(t)$ stays bounded from above as $t \rightarrow \infty$, contradicting $x_{k}(t)-x_{j}(t) \rightarrow \infty$ as $t \rightarrow \infty$. This proves Theorem 1 .

Proof of Theorem 2. The equations of motion of (1.4) are

$$
\dot{x}_{k}=y_{k} / m_{k} ; \quad \dot{y}_{k}=-\sum_{j<k} V_{k j}^{\prime}\left(x_{k}-x_{j}\right)+\sum_{j>k} V_{j k}^{\prime}\left(x_{j}-x_{k}\right) \text {. }
$$

Let $x(t)=\left(x_{1}(t), \ldots, x_{n}(t)\right)$ be a solution of (2.2). Analogously as in the proof of Theorem 1 one proves the existence of the asymptotic velocities $\dot{x}_{k}(\infty)$, of their ordering (2.1), and also that $x_{k}(t)-x_{j}(t) \rightarrow \infty$ as $t \rightarrow \infty$, for all $j<k$. Proceeding indirectly we assume that $\dot{x}_{j}(\infty)=\dot{x}_{k}(\infty)$ for some $j<k$. It is no restriction to assume (2.2). From now on we keep $j$ and $k$ fixed. Existence of the asymptotic velocities and $\dot{x}_{k}(\infty)-\dot{x}_{j}(\infty)=0$ imply that $\dot{x}_{k}(t)-\dot{x}_{j}(t)=-\int_{t}^{\infty} \ddot{x}_{k}(s)-\ddot{x}_{j}(s) d s$,
hence, using the repulsivity (A1) for each $V_{k j}$ we obtain that

$$
\begin{aligned}
& x_{k}(t)-x_{j}(t)=x_{k}(0)-x_{j}(0)-t \int_{t}^{\infty} \ddot{x}_{k}(s)-\ddot{x}_{j}(s) d s-\int_{0}^{t} s\left(\ddot{x}_{k}(t)-\ddot{x}_{j}(t)\right) d s \\
& <x_{k}(0)-x_{j}(0)-t \int_{t}^{\infty}\left(m_{k}^{-1} \sum_{r>k} V_{r k}^{\prime}\left(x_{k}(s)-x_{j}(s)\right)+m_{j}^{-1} \sum_{r<j} V_{j r}^{\prime}\left(x_{k}(s)-x_{j}(s)\right)\right) d s \\
& -\int_{0}^{t} s\left(m_{k}^{-1} \sum_{r>k} V_{r k}^{\prime}\left(x_{k}(s)-x_{j}(s)\right)+m_{j}^{-1} \sum_{r<j} V_{j r}^{\prime}\left(x_{k}(s)-x_{j}(s)\right)\right) d s .
\end{aligned}
$$

The assumption (2.2), the ordering (2.1), the condition (A4), and the statement (i) of the lemma imply that the integral $\int_{0}^{t} s(\ldots) d s$ on the right-hand side of the above inequality converges as $t \rightarrow \infty$, hence also the limit $t \int_{t}^{\infty} \ldots d s$ exists as $t \rightarrow \infty$. Therefore $x_{k}(t)-x_{j}(t)$ is bounded from above as $t \rightarrow \infty$. But this is a contradiction to $x_{k}(t)-x_{j}(t) \rightarrow \infty$ as $t \rightarrow \infty$.

\section{Discussion of Example 1}

Choose $b=\left(b_{1}, b_{2}, b_{3}\right)=(0,0,1)$. We construct now an orbit $x(t)=\left(x_{1}(t), x_{2}(t)\right.$, $\left.x_{3}(t)\right)$ for which the distance between the first two particles grows only as $\sqrt{t}$ as $t \rightarrow \infty$ and for which $\dot{x}(\infty)=b$. For $T>0$ we define the space

$$
\begin{aligned}
F_{T}= & \left\{u=\left(u_{1}, u_{2}, u_{3}\right) \in C\left([T, \infty), \mathbb{R}^{3}\right) \mid u(T)=0 ;\right. \\
& \sqrt{2 s}-\sqrt{2 T}-1 \leqq u_{2}(s)-u_{1}(s) \leqq 8 \sqrt{2 s} ; \\
& \left.\left|u_{2}(t)\right| \leqq 8 \sqrt{2 s} ;\left|u_{3}(t)\right| \leqq 8 \sqrt{2 s} \text { for all } s \geqq T\right\} .
\end{aligned}
$$

Furthermore we define for $u \in F_{T}$ the norm $\|u\|_{T}=\sup _{t \geqq T} \frac{\left|u_{1}(t)\right|+\left|u_{2}(t)\right|+\left|u_{3}(t)\right|}{\sqrt{t}}$ which makes $F_{T}$ to a complete metric space. Let us write the Hamiltonian (1.5) as $H(x, y)=\sum_{k=1}^{3} \frac{y_{k}^{2}}{2}+W(x)$. This defines $W(x)$. 
For large $T$ we can define the operator $I_{T}: u \in F_{T} \rightarrow I_{T} u$ by

$$
\left(I_{T} u\right)(t)=\int_{T}^{t}(s-T) W^{\prime}(\xi(s)+u(s)) d s+(t-T) \int_{t}^{\infty} W^{\prime}(\xi(s)+u(s)) d s \quad(t \geqq T),
$$

where $\xi(s)=(0,1+\sqrt{2 T}, s+1+\sqrt{2 T})$ and $W^{\prime}(x)$ is the gradient of $W$. For large $T, I_{T}$ leaves $F_{T}$ invariant. Moreover, if we fix an arbitrary $0<\vartheta<1$, then for large $T, I_{T}: F_{T} \rightarrow F_{T}$ is a contraction with contraction constant $\vartheta$.

Proof. Existence of $I_{T}$ and invariance of $F_{T}$ follow from the estimates $x_{1}(s)-x_{3}(s) \leqq x_{1}(s)-x_{2}(s) \leqq-\sqrt{2 s}$ and $s / 2 \leqq x_{3}(s)-x_{2}(s) \leqq 2 s$, which imply the estimates

$$
\begin{aligned}
& \int_{T}^{t}(s-T) e^{x_{1}-x_{k}} d s+(t-T) \int_{T}^{\infty} e^{x_{1}-x_{k}} d s=O\left(\sqrt{T} e^{-\sqrt{2 T}}\right) \quad \text { for } \quad k=2,3, \\
& \sqrt{2 t}-\sqrt{2} \bar{T} \leqq \int_{T}^{t}(s-T)\left|x_{3}-x_{2}\right|^{-1 / 2} d s+(t-T) \int_{t}^{\infty}\left|x_{3}-x_{2}\right|^{-1 / 2} d s \leqq 8(\sqrt{2 t}-\sqrt{2} \bar{T}) .
\end{aligned}
$$

The contraction property is implied by the following estimates: Let $u, v \in F_{T}$. Let $x(s)=\xi(s)+u(s), z(s)=\xi(s)+v(s)$. By application of the mean value theorem we obtain that

$$
\begin{aligned}
& \left|e^{x_{1}(s)-x_{k}(s)}-e^{z_{1}(s)-z_{k}(s)}\right| \leqq \sqrt{s} e^{-\sqrt{2 s}} \cdot \frac{\left|u_{1}(s)-v_{1}(s)\right|+\left|u_{k}(s)-v_{k}(s)\right|}{\sqrt{s}} \text { for } k=2,3, \\
& || x_{2}-\left.x_{3}\right|^{-1 / 2}-\left|z_{2}-z_{3}\right|^{-1 / 2} \mid \leqq \frac{6 \sqrt{2}}{s^{2}} \cdot \frac{\left|u_{2}(s)-v_{2}(s)\right|+\left|u_{3}(s)-v_{3}(s)\right|}{\sqrt{s}} \quad \text { for all } s \geqq T .
\end{aligned}
$$

Inserting these estimates into (3.1) gives us for large $T$ that

$$
\begin{aligned}
\sum_{j=1}^{3}\left|\left(I_{T} u\right)_{j}(t)-\left(I_{T} v\right)_{j}(t)\right| \leqq & C \int_{T}^{t} \frac{1}{s} \cdot \frac{\Sigma\left|u_{k}(s)-v_{k}(s)\right|}{\sqrt{s}} d s \\
& +C t \int_{t}^{\infty} \frac{1}{s^{2}} \cdot \frac{\Sigma\left|u_{k}(s)-v_{k}(s)\right|}{\sqrt{s}} d s
\end{aligned}
$$

and therefore that $\Sigma\left|\left(I_{T} u\right)_{j}(t)-\left(I_{T} v\right)_{j}(t)\right| \leqq C^{\prime} \log t\|u-v\|_{T}$ as $t \rightarrow \infty$, where $C$ and $C^{\prime}$ are constants independent on $T$. Hence for large $T$

$$
\left\|I_{T} u-I_{T} v\right\|_{T} \leqq \sup _{t \geqq T} \frac{C^{\prime} \log t}{\sqrt{t}}\|u-v\|_{T} \leqq \vartheta\|u-v\|_{T}
$$

The contraction mapping principle implies that $I_{T}$ has in $F_{T}$ a fixed point for large $T$; denote this fixed point by $u_{*}$. The function $t \rightarrow x(t) \in \mathbb{R}^{3}$ defined by $x(t)=\left(x_{1}(t), x_{2}(t), x_{3}(t)\right)=\xi(t)+u_{*}(t)$ is a solution of the Hamiltonian equations $\ddot{x}=-W^{\prime}(x)$ with $\dot{x}(\infty)=(0,0,1)$. The proof can easily be generalized to arbitrary $b=\left(b_{1}, b_{2}, b_{3}\right)$ with $b_{1}=b_{2}<b_{3}$. 


\section{Proof of Theorem 4}

The Two Particle System. In order to carry through the induction step, we need some properties of the two particle system. The two particle Hamiltonian is

$$
H(x, y)=\frac{y_{1}^{2}}{2}+\frac{y_{2}^{2}}{2}+V\left(x_{2}-x_{1}\right) .
$$

We denote by $q=x_{2}-x_{1}$ the relative motion. $q(t)$ is a solution of $\ddot{q}=-2 V^{\prime}(q)$; by (A1) it is therefore strictly convex. Because of $\dot{q}(-\infty)<0$ and $\dot{q}(\infty)=-\dot{q}(-\infty)>0$, there is exactly one $t_{*}$ such that $\dot{q}\left(t_{*}\right)=0$. Time reflection invariance implies that $q$ is symmetric with respect to $t_{*}$ where $q$ attains the global minimum. $t_{*}$ can be determined from the asymptotic velocities and phases of the two particle system: $t_{*}$ is the time $t$ at which the two asymptotic straight lines $a_{1}^{-}+b_{1}^{-} t, a_{2}^{-}+b_{2}^{-} t$ cross, hence

$$
t_{*}=\frac{a_{2}^{-}-a_{1}^{-}}{b_{1}^{-}-b_{2}^{-}} .
$$

Induction Step from $n-1$ to $n$ Particles. Denote the Hamiltonian (1.1) as

$$
H(x, y)=\sum_{k=1}^{n} \frac{y_{k}^{2}}{2}+V^{(n)}(x), \quad \text { where } \quad V^{(n)}(x)=\sum_{1 \leqq j<k \leqq n} V\left(x_{k}-x_{j}\right) .
$$

We fix asymptotic velocities $b_{1}^{-}>b_{2}^{-}>\ldots>b_{n}^{-}$and asymptotic phases $a_{1}^{-}$, $a_{2}^{-}, \ldots, a_{n-1}^{-}$. The asymptotic phase $a_{n}^{-}$of the $n^{\text {th }}$ particle is enlargened when we need.

We first make a heuristic argument, following the geometrical picture in the introduction (Sect. 1, see Fig. 2): Assume $a_{n}^{-}$to be very large. After the collision of the first $n-1$ particles, the collision of the $n^{\text {th }}$ particle with the $j^{\text {th }}$ particle by induction hypothesis, by (1.9), and by (4.1) place at time

$$
\frac{a_{n}^{-}}{b_{n-j}^{-}-b_{n}^{-}}+O(1) \quad \text { as } \quad a_{n}^{-} \rightarrow \infty \text {. }
$$

Therefore at time

$$
T_{j}=\frac{1}{2}\left(\frac{a_{n}^{-}}{b_{n-j}^{-}-b_{n}^{-}}+\frac{a_{n}^{-}}{b_{n-j+1}^{-}-b_{n}^{-}}\right)
$$

the particle $j$ is halfway between the collisions with particle $j+1$ and particle $j-1$. The definition (4.3) holds for $j=2,3, \ldots, n-1$. For convenience we also define

$$
\begin{aligned}
T_{n} & =\frac{1}{2} \frac{a_{n}^{-}}{b_{1}^{-}-b_{n}^{-}}, \\
T_{n+1} & =-a_{n}^{-}, \\
T_{1} & =2 \frac{a_{n}^{-}}{b_{n-1}^{-}-b_{n}^{-}} .
\end{aligned}
$$

$T_{n}$ is half of the collision time between particles $n$ and $n-1$; hence for large $a_{n}^{-}, T_{n}$ is much smaller than the time of collision between particles $n-1$ and $n$ but also much 
larger than the time of interaction between the first $n-1$ particles. $T_{n+1}$ is much smaller than the time of interaction between the first $n-1$ particles (for large $a_{n}^{-}$, the interaction time of the first $n-1$ particles is independent of $a_{n}^{-}$). $T_{1}$ is much larger than the time of the last collision which takes place between particles 1 and 2 .

Let $x(t)=\left(x_{1}(t), \ldots, x_{n}(t)\right)$ be the $n$-particle orbit with asymptotic velocities $b^{-}=\left(b_{1}^{-}, \ldots, b_{n}^{-}\right)$and asymptotic phases $a^{-}=\left(a_{1}^{-}, \ldots, a_{n}^{-}\right)$.

To proceed according to the geometrical picture in Sect. 1 (see Fig. 2) means the following: On the time interval $T_{n+1} \leqq t \leqq T_{n}$ we will approximate the orbit by an $n-1$ particle orbit and the $n^{\text {th }}$ particle moves free. On the intervals $T_{j+1} \leqq t \leqq T_{j}$ we will approximate by a two particle orbit for which the particles $j+1$ and $j$ interact and the other particles move free. On the intervals $t \leqq T_{n+1}$ and $t \geqq T_{1}$ we will approximate by all particles moving free.

In order to make these approximations, we need the following technical lemma. It shows that from the distance between the particles we can make conclusions on how close to the free motion the particles already are.

Lemma 1. Let $N \geqq 2$. Let $T$ be an arbitrary real number. Let $\alpha, M, R$ be the constants from the condition (B). Let $x=\left(x_{1}, \ldots, x_{N}\right), y=\left(y_{1}, \ldots, y_{N}\right)$ such that $x_{1}<x_{2}<\ldots<x_{N}, y_{1}<y_{2}<\ldots<y_{N}$, and $x_{k}-x_{j}>R$ for all $1 \leqq j<k \leqq N$. Let $\beta=\frac{1}{3} \inf _{j<k} y_{k}-y_{j}$. Let $0<\varepsilon \leqq \beta$. Then there exists a constant $C_{N}>0$ such that the following holds: If $\left(x_{k}-x_{j}\right)^{1+\alpha}>C_{N} M / \beta \varepsilon$ for all $1 \leqq j<k \leqq N$, then the velocities of the $N$ particle orbit with initial conditions $x(t)=x$, and $\dot{x}(T)=y$ are very close to $y$ for all $t \geqq T$, more precisely: $\left|\dot{x}_{k}(t)-y_{k}\right| \leqq \varepsilon$ for all $t \geqq T$ and for all $k=1, \ldots, N$.

Proof of Lemma 1. Define the space

$$
F=\left\{u \in C^{1}\left([T, \infty), \mathbb{R}^{N}\right)|u(T)=0 ; \dot{u}(T)=0 ;| \dot{u}(t) \mid \leqq \varepsilon \text { for all } t \geqq T\right\} .
$$

Define $\xi(t)=x+(t-T) y \in \mathbb{R}^{N}$. For $u \in F$ define $I u$ by

$$
(I u)(t)=-\int_{T}^{t} d s \int_{T}^{s} d \tau \operatorname{grad} V^{(N)}(\xi(\tau)+u(\tau))
$$

Define $\varrho:=\min _{j<k} x_{k}-x_{j} . \quad$ Let $u \in F . \quad$ Evidently, $\xi_{k}(t)-\xi_{j}(t)+u_{k}(t)-u_{j}(t)$ $\geqq x_{k}-x_{j}+(t-T)\left(y_{k}-y_{j}-2 \varepsilon\right)>\varrho+(t-T) \beta$ for all $t \geqq T$, hence

$$
\begin{aligned}
\left|\frac{d}{d t}(I u)(t)\right| & \leqq \int_{T}^{t}\left|\operatorname{grad} V^{(N)}(\xi(s)+u(s))\right| d s \leqq C_{N}^{\prime} \int_{T}^{\infty} \frac{M}{(\varrho+\beta \cdot(t-T))^{2+\alpha}} d s \\
& =\frac{C_{N} M}{\beta \cdot \varrho^{1+\alpha}} \leqq \varepsilon .
\end{aligned}
$$

By the Schauder fixed point theorem, $I$ has a fixed point $u_{*}$ in $F . x=\xi+u_{*}$ is the $N$ particle orbit with the right initial conditions. By construction it has the claimed properties. This proves Lemma 1.

Remark. By time inversion, an analogous version of Lemma 1 holds as time tends to minus infinity. 
Denote the asymptotic velocities and phases at plus infinity by $b^{+}$and $a^{+}$. The next lemma will give information how we can approximate the orbit $x(t)$ by the asymptotic free motions $a^{ \pm}+b^{ \pm} t$ as $t \rightarrow \pm \infty$ before the first collision and after the last collision.

Lemma 2. There exist constants $c$ and $t_{0}$ independent on $a_{n}^{-}$such that for large $a_{n}^{-}$ the following holds:

(i) $\left|x(t)-a^{-}-b^{-} t\right|<c|t|^{-\alpha},\left|\dot{x}(t)-b^{-}\right|<c|t|^{-1-\alpha}$ for all $t<-t_{0}$,

(ii) $\left|x(t)-a^{+}-b^{+} t\right|<c\left|t-a_{n}^{-} /\left(b_{n-1}^{-}-b_{n}^{-}\right)\right|^{-\alpha}$, and $\left|\dot{x}(t)-b^{+}\right|<c\left|t-a_{n}^{-} /\left(b_{n-1}^{-}-b_{n}^{-}\right)\right|^{-1-\alpha}$ for all $t>t_{0}+a_{n}^{-} /\left(b_{n-1}^{-}-b_{n}^{-}\right)$,

where $c>0$ is some constant independent of $a_{n}^{-}$and $t_{0}$.

Observe that $a_{n}^{-} /\left(b_{n}^{-}-b_{n-1}^{-}\right)$is according to (4.1) and the induction hypothesis and the heuristic argument the time of the last collision which takes place between particles 1 and 2.

Proof of Lemma 2. First we prove (i). The decay properties (B) of $V^{\prime}$ and the pairwise distinctness of the asymptotic velocities allow us to write

$$
x(t)=a^{-}+b^{-} t-\int_{-\infty}^{t}(t-s) \operatorname{grad} V^{(n)}(x(s)) d s,
$$

where the integral converges absolutely. Inspired from this integral equation we define the space

$$
F_{T}=\left\{\left.u \in C^{1}\left((-\infty,-T], \mathbb{R}^{n}\right)|| u(t)|\leqq c| t\right|^{-\alpha} \text { and }|\dot{u}(t)| \leqq c|t|^{-1-\alpha} \text { for } t \leqq-T\right\},
$$

and on it the integral operator $I_{T}$ by

$$
\left(I_{T} u\right)(t)=-\int_{-\infty}^{t}(t-s) \operatorname{grad} V^{(n)}\left(a^{-}+b^{-} s+u(s)\right) d s \text { for } t \leqq-T \quad\left(u \in F_{T}\right) .
$$

If $T$ is large, then $I_{T}$ leaves $F_{T}$ invariant and is a contraction with respect to the norm $\|u\|_{T}=\sup _{t \leqq-T} \max \{|u(t)|,|\dot{u}(t)|\}$.

Proof. Fix some contraction constant $0<\vartheta<1$. Let $u, v \in F_{T}$. Let $x(s)=a^{-}+b^{-} s+u(s), y(s)=a^{-}+b^{-} s+v(s)$. Define $B:=\min _{1 \leqq k<j \leqq n} b_{j}^{-}-b_{k}^{-}$.

If $T$ is large enough, then for all $s \leqq-T$ $x_{j}(s)-x_{k}(s) \geqq|B s| \geqq R, y_{j}(s)-y_{k}(s) \geqq|B s| \geqq R$ for all $k<j$ for large $a_{n}^{-}$, and therefore for all $t \leqq-T$,

$$
\begin{aligned}
\left|\left(I_{T} u\right)(t)\right| & \leqq 2 \sum_{k<j} \int_{-\infty}^{t}(t-s)\left|V^{\prime}\left(x_{j}(s)-x_{k}(s)\right)\right| d s \\
& \leqq n(n-1) \int_{-\infty}^{t} 2|s| M /|B s|^{2+\alpha} d s=2 n(n-1) M / \alpha B^{2+\alpha}|t|^{\alpha},
\end{aligned}
$$




$$
\begin{aligned}
\left|\left(I_{T} u\right)^{\prime}(t)\right| & \leqq \int_{-\infty}^{t}\left|\operatorname{grad} V^{(n)}(x(s))\right| d s \leqq n(n-1) / 2 \int_{-\infty}^{t} M /|B S|^{2+\alpha} d s \\
& =n(n-1) / 2 M /(1+\alpha) B^{2+\alpha}|t|^{1+\alpha},
\end{aligned}
$$

which implies the invariance of $F_{T}$ for $c=2 n(n-1) M / \alpha B^{2+\alpha}$.

The contraction property is implied by (4.6), (B) and the mean value theorem:

$$
\begin{gathered}
\left|\left(I_{T} u\right)(t)-\left(I_{T} v\right)(t)\right| \leqq \int_{t}^{\infty}(t-s)|\operatorname{grad} V(x(s))-\operatorname{grad} V(y(s))| d s \\
\leqq \int_{t}^{\infty}(t-s) M /|B s|^{3+\alpha}|u(s)-v(s)| d s \leqq \int_{t}^{\infty} 2|s| M /|B s|^{3+\alpha} d s \cdot\|u-v\|_{T} \\
=2 M /(1+\alpha) B^{3+\alpha}|t|^{1+\alpha} \cdot\|u-v\|_{T} \leqq \vartheta\|u-v\|_{T}, \\
\left|\left(I_{T} u\right)^{\prime}(t)-\left(I_{T} v\right)^{\prime}(t)\right| \leqq \int_{t}^{\infty}|\operatorname{grad} V(x(s))-\operatorname{grad} V(y(s))| d s \\
\leqq \int_{t}^{\infty} M /|B s|^{3+\alpha}|u(s)-v(s)| d s \leqq \int_{t}^{\infty} M /|B s|^{3+\alpha} d s \cdot\|u-v\|_{T} \\
=M /(2+\alpha) B^{3+\alpha}|t|^{2+\alpha} \cdot\|u-v\|_{T} \leqq \vartheta\|u-v\|_{T} \cdot
\end{gathered}
$$

This proves (i). Proof of (ii): Let $\tau=a_{n}^{-} /\left(b_{n-1}^{-}-b_{n}^{-}\right)$. We define for $T>0$ the space

$$
\begin{gathered}
F_{T}^{\prime}=\left\{u \in C^{1}\left([T+\tau, \infty), \mathbb{R}^{n}\right)|| u(t)|\leqq c| t-\left.\tau\right|^{-\alpha},\right. \\
\left.|\dot{u}(t)| \leqq c|t-\tau|^{-1-\alpha} \text { for all } t \geqq T+\tau\right\} .
\end{gathered}
$$

Furthermore we define the operator $I_{T}$ by

$$
\left(I_{T} u\right)(t)=-\int_{t}^{\infty}(s-t) \operatorname{grad} V^{(n)}\left(a^{+}+b^{+} s+u(s)\right) d s \quad \text { for } \quad t \geqq T+\tau \quad\left(u \in F_{T}^{\prime}\right) .
$$

If $T$ is large, then $I_{T}$ leaves $F_{T}^{\prime}$ invariant and is a contraction: In order to prove this, observe that about $a^{+}=\left(a_{1}^{+}, \ldots, a_{n}^{+}\right)$we know by (1.9), since $G_{n}$ is independent of $a_{n}^{-}$, that

$$
a_{1}^{+}=a_{n}^{-}+O(1), a_{k}^{+}=O(1) \quad \text { for } k=2, \ldots, n \text { as } a_{n}^{-} \rightarrow \infty .
$$

Observe furthermore that that $\tau$ is (up to $O(1)$ as $a_{n}^{-} \rightarrow \infty$ ) the time, where the two asymptotic straight lines $a_{1}^{+}+b_{1}^{+} t, a_{2}^{+}+b_{2}^{+} t$ cross. $T$ large means that we are far away from this crossing point. Therefore, by using (1.6) and (4.7), we obtain for large $T$ that

$$
\begin{aligned}
x_{k}(t)-x_{1}(t)= & \left(b_{n-k+1}^{-}-b_{n}^{-}\right) t-a_{n}^{-}+O(1)+u_{k}(t)-u_{1}(t) \\
= & \left(b_{n-k+1}^{-}-b_{n}^{-}\right) t-a_{n}^{-}+O(1) \\
= & \left(b_{n-k+1}^{-}-b_{n}^{-}\right) \cdot\left(t-a_{n}^{-} /\left(b_{n-1}^{-}-b_{n}^{-}\right)\right) \\
& +a_{n}^{-} \cdot\left(\frac{b_{n-k+1}^{-}-b_{n}^{-}}{b_{n-1}^{-}-b_{n}^{-}}-1\right)+O(1) \\
\geqq & \left(b_{n-k+1}^{-}-b_{n}^{-}\right) \cdot\left(t-a_{n}^{-} /\left(b_{n-1}^{-}-b_{n}^{-}\right)\right)+O(1),
\end{aligned}
$$


where we used that $\frac{b_{n-k+1}^{-}-b_{n}^{-}}{b_{n-1}^{-}-b_{n}^{-}} \geqq 1$ for $k=2, \ldots, n$. Furthermore for $2 \leqq j<k \leqq n$,

$$
\begin{aligned}
x_{k}(t)-x_{j}(t) & =\left(b_{n-k+1}^{-}-b_{n-j+1}^{-}\right) t+O(1)+u_{k}(t)-u_{j}(t) \\
& =\left(b_{n-k+1}^{-}-b_{n-j+1}^{-}\right) t+O(1) \\
& \geqq\left(b_{n-k+1}^{-}-b_{n-j+1}^{-}\right) \cdot\left(t-a_{n}^{-} /\left(b_{n-1}^{-}-b_{n}^{-}\right)\right)+O(1) .
\end{aligned}
$$

Let $B=\frac{1}{2} \inf _{j<i} b_{j}^{-}-b_{i}^{-}$. Then the above estimates imply that

$$
x_{k}(t)-x_{j}(t) \geqq B(t-\tau)+O(1) \geqq R \quad \text { for all } 1 \leqq j<k \leqq n
$$

for all $t \geqq T+\tau$, where the $O(1)$ terms are uniform in $a_{n}^{-}$and in $t \geqq T$. The remaining estimates to prove invariance of $F_{T}^{\prime}$ and the contraction property are analogous to those in the proof of (i). This proves (ii).

We now define the following vectors of $\mathbb{R}^{n}$ :

$$
\begin{aligned}
B_{n+1}= & \left(b_{1}^{-}, b_{2}^{-}, \ldots, b_{n-1}^{-}, b_{n}^{-}\right)=b^{-}, A_{n+1}=\left(a_{1}^{-}, a_{2}^{-}, \ldots, a_{n-1}^{-}, a_{n}^{-}\right)=a^{-}, \\
B_{n}= & \left(b_{n-1}^{-}, b_{n-2}^{-}, \ldots, b_{2}^{-}, b_{1}^{-}, b_{n}^{-}\right), \\
A_{n}= & \left(a_{n-1}^{-}+\frac{\partial G_{n-1}}{\partial b_{1}^{-}}, \ldots, a_{2}^{-}+\frac{\partial G_{n-1}}{\partial b_{n-2}^{-}}, a_{1}^{-}+\frac{\partial G_{n-1}}{\partial b_{n-1}^{-}}, a_{n}^{-}\right), \\
B_{j}= & \left(b_{n-1}^{-}, b_{n-2}^{-}, \ldots, b_{n-j+1}^{-}, b_{n}^{-}, b_{n-j}^{-}, \ldots, b_{2}^{-}, b_{1}^{-}\right), \\
A_{j}= & \left(a_{n-1}^{-}+\delta_{1}^{(n-1)}, \ldots, a_{n-j+1}^{-}+\delta_{j-1}^{(n-1)}, a_{n}^{-}+\sum_{k=1}^{n-j} \delta\left(b_{n}^{-}-b_{k}^{-}\right),\right. \\
& \left.a_{n-j}^{-}+\delta_{j}^{(n-1)}-\delta\left(b_{n}^{-}-b_{n-j}^{-}\right), \ldots, a_{1}^{-}+\delta_{n-1}^{(n-1)}-\delta\left(b_{n}^{-}-b_{1}^{-}\right)\right) \\
& \text {for } j=1, \ldots, n-1 .
\end{aligned}
$$

Observe that $B_{1}=b^{+}$. We have to prove that $A_{1}=a^{+}$. The following lemma describes the collision of the first $n-1$ particles.

Lemma 3. The following statements hold: $\dot{x}\left(T_{n+1}\right)=b^{-}+O\left(\left(a_{n}^{-}\right)^{-1-\alpha}\right)$ and $x\left(T_{n+1}\right)-T_{n+1} \dot{x}\left(T_{n+1}\right)=a^{-}+O\left(\left(a_{n}^{-}\right)^{-\alpha}\right)$ as $a_{n}^{-} \rightarrow \infty$. Furthermore there exists a solution $X(t)=\left(X_{1}(t), \ldots, X_{n}(t)\right)$ of the system

$$
\left\{\begin{array}{cl}
\left(\ddot{X}_{1}, \ldots, \ddot{X}_{n-1}\right) & =-\operatorname{grad} V^{(n-1)}\left(X_{1}, \ldots, X_{n-1}\right) \\
\ddot{X}_{n} & =0
\end{array}\right.
$$

such that $x-X=O\left(\left(a_{n}^{-}\right)^{-\alpha}\right), \dot{x}-\dot{X}=O\left(\left(a_{n}^{-}\right)^{-1-\alpha}\right)$ as $a_{n}^{-} \rightarrow \infty$, uniformly on $T_{n+1} \leqq t \leqq T_{n}$. Consequently, by induction hypothesis,

$$
\dot{x}\left(T_{n}\right)=B_{n}+O\left(\left(a_{n}^{-}\right)^{-1-\alpha}\right), \quad x\left(T_{n}\right)-T_{n} \dot{x}\left(T_{n}\right)=A_{n}+o(1) \quad \text { as } \quad a_{n}^{-} \rightarrow \infty .
$$

Proof of Lemma 3. Let $\bar{X}(t)=\left(\bar{X}_{1}(t), \ldots, \bar{X}_{n}(t)\right)$ be the solution of (4.8) with the same asymptotic data $a^{-}, b^{-}$as $x(t)$, i.e. $\bar{X}(t)=a^{-}+b^{-} t+O\left(|t|^{-\alpha}\right)$, $\bar{X}(t)=b^{-}+O\left(|t|^{-1-\alpha}\right)$ as $t \rightarrow-\infty$. Observe that $\bar{X}_{1}(t), \ldots, \bar{X}_{n-1}(t)$ are independent of $a_{n}^{-}$. So by Lemma 2 (i) there exists $t^{\prime}$ independent of $a_{n}^{-}$such that 
for all $t \leqq t^{\prime}$,

$\dot{x}_{j}(t)-\dot{x}_{k}(t) \geqq B ; \quad x_{j}(t)-x_{k}(t) \geqq R+B\left|t-t^{\prime}\right| \quad$ for $\quad 1 \leqq j<k \leqq n-1 ;$

$x_{k}(t)-x_{n}(t) \geqq a_{n}^{-}+C+B\left|t-t^{\prime}\right| \quad$ for $k=1, \ldots, n-1$,

$\bar{X}_{j}(t)-\bar{X}_{k}(t) \geqq B ; \quad \bar{X}_{j}(t)-\bar{X}_{k}(t) \geqq R+B\left|t-t^{\prime}\right| \quad$ for $1 \leqq j<k \leqq n-1$;

$\bar{X}_{k}(t)-\bar{X}_{j}(t) \geqq a_{n}^{-}+C+B\left|t-t^{\prime}\right| \quad$ for $k=1, \ldots, n-1$,

and furthermore

$$
\left.\begin{array}{l}
\bar{X}_{j}\left(t^{\prime}\right)-\bar{X}_{k}\left(t^{\prime}\right)>R \\
\left(\bar{X}_{j}\left(t^{\prime}\right)-\bar{X}_{k}\left(t^{\prime}\right)\right)^{1+\alpha}>4 C_{n-1} / B^{2}
\end{array}\right\} \quad \text { for } 1 \leqq k<j \leqq n-1,
$$

where $B=1 / 2 \inf _{1 \leqq k<j \leqq n} b_{j}^{-}-b_{k}^{-}, C$ is some constant independent of $a_{n}^{-}$, and $C_{n-1}$ is the constant $C_{N}$ from Lemma 1 with $N=n-1$. Fix $\varepsilon>0$ small. Then furthermore, by using the induction hypothesis, there exists $t^{\prime \prime}$ independent of $a_{n}^{-}$ such that

$$
\left.\begin{array}{l}
\bar{X}_{j}\left(t^{\prime \prime}\right)-\bar{X}_{k}\left(t^{\prime \prime}\right)>R \\
\left(\bar{X}_{j}\left(t^{\prime \prime}\right)-\bar{X}_{k}\left(t^{\prime \prime}\right)\right)^{1+x}>4 C_{n-1} / B^{2}
\end{array}\right\} \quad \text { for } \quad 1 \leqq k<j \leqq n-1,
$$

$\dot{\bar{X}}_{j}\left(t^{\prime \prime}\right)-\dot{\bar{X}}_{k}\left(t^{\prime \prime}\right)>B ; \quad \bar{X}_{j}\left(t^{\prime \prime}\right)-\bar{X}_{k}\left(t^{\prime \prime}\right)>B t^{\prime \prime} \quad$ for $1 \leqq k<j \leqq n-1$ $\dot{\bar{X}}_{n}\left(t^{\prime \prime}\right)-\dot{\bar{X}}_{k}\left(t^{\prime \prime}\right)<-\left(b_{1}^{-}-b_{n}^{-}\right)+\varepsilon ; \bar{X}_{n}\left(t^{\prime \prime}\right)-\bar{X}_{k}\left(t^{\prime \prime}\right) \geqq a_{n}^{-}+C$ for $k<n$.

We now show that the orbits $x(t)$ and $\bar{X}(t)$ are close together on $-\infty<t<t^{\prime \prime}$ as $a_{n}^{-}$is large. Using $x(t)-\bar{X}(t)=\int_{-\infty}^{t}(t-s)(\ddot{x}(s)-\ddot{X}(s)) d s$, we obtain that

$$
\begin{aligned}
|x(t)-\bar{X}(t)| \leqq & \int_{-\infty}^{t}(t-s)\left|\operatorname{grad} V^{(n-1)}(x(s))-\operatorname{grad} V^{(n-1)}(\bar{X}(s))\right| d s \\
& +\sum_{k=1}^{n-1} \int_{-\infty}^{t}(t-s)\left|V^{\prime}\left(x_{k}(s)-x_{n}(s)\right)\right| d s .
\end{aligned}
$$

Application of (4.9) and the mean value theorem give us

$$
|x(t)-\bar{X}(t)| \leqq \int_{-\infty}^{t}(t-s) f(s)|x(t)-\bar{X}(t)| d s+O\left(\left(a_{n}^{-}\right)^{-\alpha}\right),
$$

where

$$
f(s)=\left\{\begin{array}{cl}
\frac{c_{1} M}{\left(R+B\left|s-t^{\prime}\right|\right)^{3+\alpha}} & \text { for } t \leqq t^{\prime} \\
c_{1} c_{2} & \text { for } t^{\prime}<t \leqq t^{\prime \prime},
\end{array}\right.
$$

where $c_{1}$ and $c_{2}$ both are constants independent of $a_{n}^{-}\left(c_{1}\right.$ depends on the number of particles and the choice of the norm; $c_{2}$ is an upper bound on $V^{\prime \prime}$ which is determined by energy conservation, (A3), and (B)). By application of the Gronwall inequality we obtain that

$$
x(t)-\bar{X}(t)=O\left(\left(a_{n}^{-}\right)^{-\alpha}\right) \quad \text { uniformly on }-\infty<t \leqq t^{\prime \prime} .
$$



Applying analogous arguments to the equation $\dot{x}(t)-\dot{\bar{X}}(t)=\int_{-\infty}^{t} \ddot{x}(s)-\ddot{X}(s) d s$
gives

$$
|\dot{X}(t)-\dot{\bar{X}}(t)| \leqq \int_{-\infty}^{t} f(s)|x(s)-\bar{X}(s)| d s+O\left(\left(a_{n}^{-}\right)^{-1-\alpha}\right) \quad \text { for all } t \leqq t^{\prime \prime} .
$$

Inserting (4.13a) into this inequality yields

$$
\dot{x}(t)-\dot{X}(t)=O\left(\left(a_{n}^{-}\right)^{-\alpha}\right) \quad \text { uniformly on }-\infty<t \leqq t^{\prime \prime} .
$$

We now choose $X(t)$ to be the orbit of (4.8) with initial conditions

$$
X\left(t^{\prime}\right)=x\left(t^{\prime}\right), \quad \dot{X}\left(t^{\prime}\right)=\dot{x}\left(t^{\prime}\right) .
$$

We have to estimate the differences $X-x$ and $\dot{X}-\dot{x}$ on the time interval $\left[T_{n+1}, T_{n}\right]$. For this purpose we write $\left[T_{n+1}, T_{n}\right]=\left[T_{n+1}, t^{\prime}\right] \cup\left[t^{\prime}, t^{\prime \prime}\right] \cup\left[t^{\prime \prime}, T_{n}\right]$, i.e. we divide $\left[T_{n+1}, T_{n}\right]$ into three subintervals. We will make on each subinterval different estimates.

We first prove that $X-x=O\left(\left(a_{n}^{-}\right)^{-\alpha}\right), \dot{X}-\dot{x}=O\left(\left(a_{n}^{-}\right)^{-1-\alpha}\right)$ uniformly on $\left[T_{n+1}, t^{\prime}\right]$. Equations (4.10) and (4.13) imply that for large $a_{n}^{-}$

$$
\left.\begin{array}{l}
X_{j}\left(t^{\prime}\right)-X_{k}\left(t^{\prime}\right)>R \\
\left(X_{j}\left(t^{\prime}\right)-X_{k}\left(t^{\prime}\right)\right)^{1+\alpha}>4 C_{n-1} / B^{2}
\end{array}\right\} \quad \text { for } 1 \leqq k<j \leqq n-1 .
$$

Therefore the assumptions of Lemma 1 are satisfied with $\varepsilon=\frac{B}{4}$ and we conclude that for all $t \leqq t^{\prime}$,

$$
\begin{array}{ll}
\dot{X}_{j}(t)-\dot{X}_{k}(t) \geqq \frac{B}{2} ; \quad X_{j}(t)-X_{k}(t) \geqq R+\frac{B}{2}\left|t-t^{\prime}\right| & \text { for } 1 \leqq k<j \leqq n-1, \\
X_{k}(t)-X_{n}(t) \geqq a_{n}^{-}+C+\frac{B}{2}\left|t-t^{\prime}\right| & \text { for } k=1, \ldots, n-1, \\
\dot{X}_{k}(t)-\dot{X}_{n}(t) \geqq \frac{B}{2} & \text { for } k=1, \ldots, n-1 .
\end{array}
$$

Application of the mean value theorem and of (B) give then for all $t \leqq t^{\prime}$,

$$
\begin{aligned}
|\dot{x}(t)-\dot{X}(t)|= & \left|\int_{t^{\prime}}^{t} \ddot{x}(s)-\ddot{X}(s) d s\right| \\
\leqq & \int_{t}^{t^{\prime}}\left|\operatorname{grad} V^{(n-1)}(x(s))-\operatorname{grad} V^{(n-1)}(X(s))\right| d s \\
& +2 \sum_{j<n} \int_{t}^{t^{\prime}}\left|V^{\prime}\left(x_{n}(s)-x_{j}(s)\right)\right| d s \\
\leqq & \int_{t}^{t^{\prime}} M /\left(R+\frac{B}{2}\left|s-t^{\prime}\right|\right)^{3+\alpha}|x(s)-X(s)| d s+O\left(\left(a_{n}\right)^{-1-\alpha}\right),
\end{aligned}
$$

hence

$$
\sup _{\left[t, t^{\prime}\right]}|\dot{x}-\dot{X}| \leqq O\left(\left(a_{n}\right)^{-1-\alpha}\right)+\int_{t}^{t^{\prime}}\left|s-t^{\prime}\right| M /\left(R+\frac{B}{2}\left|s-t^{\prime}\right|\right)^{3+\alpha} \sup _{\left[s, t^{\prime}\right]}|\dot{x}-\dot{X}| d s .
$$


Hence by the Gronwall inequality

$$
\sup _{\left[t, t^{\prime}\right]}|\dot{x}-\dot{X}| \leqq O\left(\left(a_{n}\right)^{-1-\alpha}\right) \exp \int_{t}^{t^{\prime}}\left|s-t^{\prime}\right| M /\left(R+\frac{B}{2}\left|s-t^{\prime}\right|\right)^{3+\alpha} d s .
$$

The integral on the right-hand side of this inequality converges as $t \rightarrow-\infty$, hence we obtain $\dot{x}(t)-\dot{X}(t)=O\left(\left(a_{n}^{-}\right)^{-1-\alpha}\right)$ uniformly on $-\infty<t \leqq t^{\prime}$. By (4.14) and $t^{\prime}-T_{n}=O\left(a_{n}^{-}\right)$, we also obtain that $x(t)-X(t)=O\left(\left(a_{n}^{-}\right)^{-\alpha}\right)$ uniformly on $T_{n} \leqq t \leqq t^{\prime}$, as $a_{n}^{-} \rightarrow \infty$.

We next prove estimates $x(t)-X(t)=O\left(\left(a_{n}^{-}\right)^{-2-\alpha}\right), \quad \dot{x}(t)-\dot{X}(t)$ $=O\left(\left(a_{n}^{-}\right)^{-2-\alpha}\right)$ on the time interval $t^{\prime} \leqq t \leqq t^{\prime \prime}$ : By the mean value theorem,

$$
|\dot{x}(t)-\dot{X}(t)| \leqq \int_{t^{\prime}}^{t} c|x(s)-X(s)| d s+2 \sum_{j<n} \int_{t^{\prime}}^{t}\left|V^{\prime}\left(x_{n}(s)-x_{j}(s)\right)\right| d s,
$$

where $c$ is an upper bound on the second derivatives of $V^{(n-1)}$ on an appropriate subset of the phase space. Since $t^{\prime \prime}-t^{\prime}=O(1)$ as $a_{n}^{-} \rightarrow \infty$, the upper inequality yields

$$
\sup _{\left[t^{\prime}, t\right]}|\dot{x}-\dot{X}| \leqq \int_{t^{\prime}}^{t} c\left|s-t^{\prime}\right| \sup _{\left[t^{\prime}, s\right]}|\dot{x}-\dot{X}| d s+O\left(\left(a_{n}^{-}\right)^{-2-\alpha}\right),
$$

which implies by Gronwall inequality, since $t^{\prime \prime}-t^{\prime}$ is bounded, that $\dot{x}-\dot{X}=O\left(\left(a_{n}^{-}\right)^{-2-\alpha}\right)$. This implies by (4.14) and the boundedness of $t^{\prime \prime}-t^{\prime}$, that $x-X=O\left(\left(a_{n}^{-}\right)^{-2-\alpha}\right)$ uniformly on $t^{\prime \prime} \leqq t \leqq t^{\prime}$.

Next we prove the estimates $\left.x-\bar{X}=\bar{O}\left(a_{n}^{-}\right)^{-\alpha}\right), \dot{x}-\dot{X}=O\left(\left(a_{n}^{-}\right)^{-1-\alpha}\right)$ on the time interval $t^{\prime \prime} \leqq t \leqq T_{n}$ : By (4.11), (4.13), and $X\left(t^{\prime \prime}\right)-x\left(t^{\prime \prime}\right)=O\left(\left(a_{n}^{-}\right)^{-2-\alpha}\right)$ we can conclude that $X\left(t^{\prime \prime}\right)$ satisfies (4.11) and $X_{j}\left(t^{\prime \prime}\right)-X_{k}\left(t^{\prime \prime}\right) \geqq B$. Therefore the assumptions of Lemma 1 are satisfied with $\varepsilon=\frac{B}{2}$ and we conclude by Lemma 1 that for all $t \geqq t^{\prime \prime}$,

$$
\dot{X}_{j}(t)-\dot{X}_{k}(t) \geqq \frac{B}{2} ; \quad X_{j}(t)-X_{k}(t) \geqq R+\frac{B}{2}\left(t-t^{\prime \prime}\right) \quad \text { for } \quad 1 \leqq k<j \leqq n-1 .
$$

We need an analogous estimate on $x(t)$ in order to apply the Gronwall inequality. By the following fixed point argument we will obtain such estimates.

We define the space

$$
F_{\varepsilon}=\left\{u \in C^{1}\left(\left[t^{\prime \prime}, T_{n}\right], \mathbb{R}^{n}\right)\left|u\left(t^{\prime \prime}\right)=0, \dot{u}\left(t^{\prime \prime}\right)=0,\right| \dot{u}(t) \mid \leqq \varepsilon \quad \text { on } t^{\prime \prime} \leqq t \leqq T_{n}\right\}
$$

with the metric $\|u-v\|_{F_{\varepsilon}}=\sup _{\left[t^{\prime}, T_{n}\right]}|\dot{u}(t)-\dot{v}(t)|$. On $F_{\varepsilon}$ we define the operator $I$ by

$$
(I u)(t)=-\int_{t^{\prime \prime}}^{t} d s \int_{t^{\prime \prime}}^{s} d \tau \operatorname{grad} V^{(n)}(\xi(\tau)+u(\tau)) \quad\left(u \in F_{\varepsilon}\right),
$$

where

$$
\xi(\tau)=x\left(t^{\prime \prime}\right)+\left(\tau-t^{\prime \prime}\right) \cdot x\left(t^{\prime \prime}\right) .
$$


For small $\varepsilon, I$ leaves $F_{\varepsilon}$ invariant, which follows from the following estimates: Let $u \in F_{\varepsilon}$ and $z=\xi+u$. Then for small $\varepsilon$ by (4.12),

$$
\begin{array}{ll}
z_{j}(t)-z_{k}(t)>B t & \text { for all } 1 \leqq k< \\
z_{n}(t)-z_{k}(t)>a_{n}^{-}+O(1)-\bar{B}\left(t-t^{\prime \prime}\right) & \text { for all } k<n,
\end{array}
$$

where $\bar{B}=b_{1}^{-}-b_{n}^{-}-3 \varepsilon$. Observe that for $\varepsilon$ small

$$
z_{n}(t)-z_{k}(t)>a_{n}^{-}+O(1)-\bar{B} T_{n}>a_{n}^{-} \cdot\left(1-\frac{b_{1}^{-}-b_{n}^{-}-3 \varepsilon}{2\left(b_{1}^{-}-b_{n}^{-}\right)}\right)+O(1)>a_{n}^{-} / 4
$$

Therefore uniformly on $t^{\prime \prime} \leqq t \leqq T_{n}$.

$$
\begin{aligned}
\left|\frac{d}{d t}(I u)(t)\right| \leqq & \int_{t^{\prime \prime}}^{t} d s\left|\operatorname{grad} V^{(n-1)}(\xi(s)+u(s))\right| \\
& +2 \sum_{j<n} \int_{t^{\prime \prime}}^{t} d s\left|V^{\prime}\left(\xi_{n}(s)+u_{n}(s)-\xi_{j}(s)-u_{j}(s)\right)\right| \leqq \int_{t^{\prime \prime}}^{t} d s M /|B s|^{2+\alpha} \\
& +O\left(a_{n}^{-}\right) \cdot O\left(\left(a_{n}^{-}\right)^{-2-\alpha}\right) \leqq O\left(\left(t^{\prime \prime}\right)^{-1-\alpha}\right)+O\left(\left(a_{n}^{-}\right)^{-1-\alpha}\right),
\end{aligned}
$$

hence $\left|\frac{d}{d t}(I u)(t)\right| \leqq \varepsilon$ if $t^{\prime \prime}$ has been chosen large enough and $a_{n}^{-}$is large, which means that $F_{\varepsilon}$ is invariant under $I$. Furthermore $I$ is continuous with respect to $\|\cdot\|_{F_{\varepsilon}} \cdot F_{\varepsilon}$ is a compact and convex subset of a Banach space, hence by the Schauder fixed point theorem $I$ has in $F_{\varepsilon}$ a fixed point $u_{*} \cdot \xi+u_{*}$ is a solution of (1.2) with initial condition $\xi\left(t^{\prime \prime}\right)+u_{*}\left(t^{\prime \prime}\right)=x\left(t^{\prime \prime}\right), \dot{\xi}\left(t^{\prime \prime}\right)+\dot{u}_{*}\left(t^{\prime \prime}\right)=\dot{x}\left(t^{\prime \prime}\right)$. Since the solutions of (1.2) are unique, we know now that $x-\xi=u_{*} \in F_{\varepsilon} . x-\xi \in F_{\varepsilon}$ implies by (4.12) and (4.13b) for small $\varepsilon$ that

$$
\begin{array}{ll}
x_{j}(t)-x_{k}(t) \geqq R+B\left(t-t^{\prime \prime}\right) \geqq R & \text { for } 1 \leqq k<j \leqq n-1, \\
x_{n}(t)-x_{k}(t) \geqq a_{n}^{-}+O(1)-\bar{B}\left(t-t^{\prime \prime}\right) \geqq R & \text { for } k=1, \ldots, n-1,
\end{array}
$$

hence, by using $x\left(t^{\prime \prime}\right)-X\left(t^{\prime \prime}\right)=O\left(\left(a_{n}^{-}\right)^{-2-\alpha}\right), \dot{x}\left(t^{\prime \prime}\right)-\dot{X}\left(t^{\prime \prime}\right)=O\left(\left(a_{n}^{-}\right)^{-2-\alpha}\right)$, and $T_{n}-t^{\prime \prime}=O\left(a_{n}^{-}\right)$,

$$
\begin{aligned}
|\dot{x}(t)-\dot{X}(t)| \leqq & \left|\dot{x}\left(t^{\prime \prime}\right)-\dot{X}\left(t^{\prime \prime}\right)\right|+2 \sum_{j<n} \int_{t^{\prime \prime}}^{t}\left|V^{\prime}\left(x_{n}(s)-x_{j}(s)\right)\right| d s \\
& +\int_{t^{\prime \prime}}^{t}\left|\operatorname{grad} V^{(n-1)}(x(s))-\operatorname{grad} V^{(n-1)}(X(s))\right| d s \\
\leqq & O\left(\left(a_{n}^{-}\right)^{-2-\alpha}\right)+2 \sum_{j<n} \int_{t^{\prime \prime}}^{t} O\left(\left(a_{n}^{-}\right)^{-2-x}\right) d s \\
& +\int_{t^{\prime \prime}}^{t} M /\left(B\left(s-t^{\prime \prime}\right)\right)^{3+\alpha}|x(s)-X(s)| d s \\
\leqq & O\left(\left(a_{n}^{-}\right)^{-2-\alpha}\right)+O\left(a_{n}^{-}\right) \cdot O\left(\left(a_{n}^{-}\right)^{-2-\alpha}\right) \\
& +\int_{t^{\prime \prime}}^{t} M /\left(B\left(s-t^{\prime \prime}\right)\right)^{3+\alpha}\left(\left|x\left(t^{\prime \prime}\right)-X\left(t^{\prime \prime}\right)\right|+\left(s-t^{\prime \prime}\right) \cdot \sup |\dot{x}-\dot{X}|\right) d s \\
\leqq & O\left(\left(a_{n}^{-}\right)^{-1-\alpha}\right)+\int_{t^{\prime \prime}}^{t} M\left(s-t^{\prime \prime}\right) /\left(B\left(s-t^{\prime \prime}\right)\right)^{3+\alpha} \quad \sup _{\left[t^{\prime \prime}, s\right]}|\dot{x}(s)-\dot{X}(s)| d s .
\end{aligned}
$$


As earlier, we obtain by the Gronwall inequality that

$$
\sup _{\left[t^{\prime}, t\right]}|\dot{x}-\dot{X}| \leqq O\left(\left(a_{n}^{-}\right)^{-1-\alpha}\right) \cdot \exp \int_{t^{\prime \prime}}^{t} M\left(s-t^{\prime \prime}\right) /\left(B+\left(s-t^{\prime \prime}\right)\right)^{3+\alpha} d s .
$$

Hence since the integral on the right-hand side of this inequality converges as $t \rightarrow-\infty, \quad \dot{x}(t)-\dot{X}(t)=O\left(\left(a_{n}^{-}\right)^{-1-\alpha}\right) \quad$ uniformly on $t^{\prime \prime} \leqq t \leqq T_{n}$. Since $x\left(t^{\prime \prime}\right)-X\left(t^{\prime \prime}\right)$ and $\dot{x}\left(t^{\prime \prime}\right)-\dot{X}\left(t^{\prime \prime}\right)$ both are $O\left(\left(a_{n}^{-}\right)^{-2-\alpha}\right)$, and $T_{n}-t^{\prime \prime}=O\left(a_{n}^{-}\right)$, this implies that $x(t)-X(t)=O\left(\left(a_{n}^{-}\right)^{-\alpha}\right)$, uniformly on $t^{\prime \prime} \leqq t \leqq T_{n}$. This proves Lemma 3.

The next lemma describes the two particle collisions:

Lemma 4. Fix $j \in\{2, \ldots, n-1, n\}$. Then the following holds:

$\dot{x}\left(T_{j}\right)=B_{j}+O\left(\left(a_{n}^{-}\right)^{-1-\alpha}\right), x\left(T_{j}\right)-T_{j} \dot{x}\left(T_{j}\right)=A_{j}+o(1)$ as $a_{n}^{-} \rightarrow \infty$.

Furthermore, there exists a solution $X(t)=\left(X_{1}(t), \ldots, X_{n}(t)\right)$ of the system

$$
\left\{\begin{array}{c}
\left(\ddot{X}_{j-1}, \ddot{X}_{j}\right)=-\operatorname{grad} V^{(2)}\left(X_{j-1}, X_{j}\right) \\
\ddot{X}_{k}=0 \quad \text { for } k=1, \ldots, j-2, j+1, \ldots, n
\end{array}\right.
$$

such that $x-X=O\left(\left(a_{n}^{-}\right)^{-\alpha}\right), \dot{x}-\dot{X}=O\left(\left(a_{n}^{-}\right)^{-1-\alpha}\right)$ as $a_{n}^{-} \rightarrow \infty$, uniformly on $T_{j} \leqq t \leqq T_{j-1}$. Consequently, by the scattering properties of the two particle system, $\dot{x}\left(T_{j-1}\right)=B_{j-1}+O\left(\left(a_{n}^{-}\right)^{-1-\alpha}\right), x\left(T_{j-1}\right)-T_{j-1} \dot{x}\left(T_{j-1}\right)=A_{j-1}+o(1)$ as $a_{n}^{-} \rightarrow \infty$.

Proof of Lemma 4. The proof is by induction $j \rightarrow j-1$. Fix $j<n$. As induction hypothesis, we assume (4.15) to be valid (for $j=n-1$, the validity of (4.15) follows from Lemma 3).

Define $\xi(t)=x\left(T_{j}\right)+\left(t-T_{j}\right) \cdot x\left(T_{j}\right)$ to be the tangent to $x$ at time $T_{j}$. Let

$$
t_{j}=\frac{a_{n}^{-}}{b_{n-j+1}^{-}-b_{n}^{-}}
$$

$t_{j}$ is up to $O(1)$ the time, where heuristically the particles $j$ and $j-1$ interact. Fix $\varepsilon>0$ small. We shall prove now that $|x(t)-\xi(t)| \leqq \varepsilon$ and $|\dot{x}(t)-\dot{\xi}(t)| \leqq \varepsilon$ for $T_{j} \leqq t \leqq t_{j}+O(1)$. For this purpose we define the space $F_{\varepsilon, T}$ and the operator $I$ by

$$
\begin{gathered}
F_{\varepsilon, T}=\left\{u \in C^{1}\left(\left[T_{j}, t_{j}-T\right], \mathbb{R}^{n}\right)\left|u\left(T_{j}\right)=0, \dot{u}\left(T_{j}\right)=0,\right| u(t) \mid \leqq \varepsilon\right. \\
\left.\quad \text { and }|\dot{u}(t)| \leqq \varepsilon \text { for } T_{j} \leqq t \leqq t_{j}-T\right\}, \\
(I u)(t)=-\int_{T_{j}}^{t} d s \int_{T_{j}}^{s} d \tau \operatorname{grad} V^{(n)}(\xi(\tau)+u(\tau)) \quad\left(u \in F_{\varepsilon, T}\right) .
\end{gathered}
$$

Claim. There exists $T_{*}$ independent of $a_{n}^{-}$such that $F_{\varepsilon, T}$ is invariant under $I$ for all $T>T_{*}$.

Proof. Let $u \in F_{\varepsilon, T}$ and $z(t)=\xi(t)+u(t)$.

By (4.15), $\xi(t)=A_{j}+t B_{j}+o(1)$ as long as $t-T_{j}=O\left(a_{n}^{-}\right)$. Therefore

$$
z_{j}(t)-z_{j-1}(t)=a_{n}^{-}-t\left(b_{n-j+1}^{-}-b_{n}^{-}\right)+O(1) .
$$


For $t=t_{j}-T$, where $T>0$, we obtain that

$$
\begin{aligned}
z_{j}\left(t_{j}-T\right)-z_{j-1}\left(t_{j}-T\right) & =a_{n}^{-}-t_{j}\left(b_{n-j+1}^{-}-b_{n}^{-}\right)+T\left(b_{n-j+1}^{-}-b_{n}^{-}\right)+O(1) \\
& =T\left(b_{n-j+1}^{-}-b_{n}^{-}\right)+O(1) \quad \text { as } \quad a_{n}^{-} \rightarrow \infty .
\end{aligned}
$$

Let $1 \leqq k<r \leqq n$. Only for $r=j$ is $\dot{\xi}_{r}-\dot{\xi}_{k}<0$; for these $k<r$ is

$$
\begin{aligned}
z_{j}(t)-z_{k}(t) & \geqq a_{n}^{-}-t\left(b_{n-k}^{-}-b_{n}^{-}\right)+O(1) \geqq a_{n}^{-}-t_{j}\left(b_{n-k}^{-}-b_{n}^{-}\right)+O(1) \\
& \geqq a_{n}^{-} \cdot \frac{b_{n-j+1}^{-}-b_{k}^{-}}{b_{n-j+1}^{-}-b_{n}^{-}}+O(1) .
\end{aligned}
$$

For all $1 \leqq k<r \leqq n$ with $r \neq j$ is $\dot{\xi}_{r}-\dot{\xi}_{k}>0$ and therefore

$$
z_{r}(t)-z_{k}(t) \geqq z_{r}\left(T_{j}\right)-z_{k}\left(T_{j}\right) \geqq T_{j} B+O(1) \geqq a_{n}^{-} \cdot \frac{B}{b_{1}^{-}-b_{n}^{-}}+O(1),
$$

where $B=\inf _{l<i} b_{i}^{-}-b_{l}^{-}$. Therefore

$$
\begin{gathered}
\int_{T_{J}}^{t} d s \int_{T_{j}}^{s} d \tau\left|V^{\prime}\left(z_{j}(\tau)-z_{j-1}(\tau)\right)\right| \leqq \int_{T_{j}}^{t} d s \int_{T_{j}}^{s} d \tau M /\left(\xi_{j}(\tau)-\xi_{j-1}(\tau)-2 \varepsilon\right)^{2+\alpha} \\
\quad \leqq \frac{M}{\alpha(1+\alpha)\left(x_{j}\left(T_{j}\right)-x_{j-1}\left(T_{j}\right)\right)^{2}} \cdot \frac{1}{\left(T\left(b_{n-j+1}^{-}-b_{n}^{-}\right)+O(1)\right)^{\alpha}}=O\left(T^{-\alpha}\right)
\end{gathered}
$$

and for all $k<r$ with $(k, r) \neq(j-1, j)$

$$
\int_{T_{J}}^{t} d s \int_{T_{J}}^{s} d \tau\left|V^{\prime}\left(z_{r}(\tau)-z_{k}(\tau)\right)\right| \leqq O\left(\left(a_{n}^{-}\right)^{-\alpha}\right) .
$$

Therefore

$$
|(I u)(t)| \leqq \int_{T_{J}}^{t} d s \int_{T_{J}}^{s} d \tau\left|\operatorname{grad} V^{(n)}(\xi(\tau)+u(\tau))\right| \leqq O\left(T^{-\alpha}\right)+O\left(\left(a_{n}^{-}\right)^{-\alpha}\right) .
$$

By analogous arguments we obtain that

$$
\left|\frac{d}{d t}(I u)(t)\right| \leqq \int_{T_{J}}^{t} d s\left|\operatorname{grad} V^{(n)}(\xi(s)+u(s))\right| \leqq O\left(T^{-1-\alpha}\right)+O\left(\left(a_{n}^{-}\right)^{-1-\alpha}\right) .
$$

Hence for $T>T_{*}$, where $T_{*}$ is independent of $a_{n}^{-}$, and large $a_{n}^{-}$we have $|(I u)(t)| \leqq \varepsilon$ and $\left|\frac{d}{d t}(I u)(t)\right| \leqq \varepsilon$, which means that $F_{\varepsilon, T}$ is invariant under $I$.

Let $T>T_{*}$. The smoothness of the potential $V$ implies that $I$ is continuous with respect to the metric $d(u, v)=\sup _{\left[T_{j}, t_{j}-T\right]}\{|u(t)-v(t)|,|\dot{u}(t)-\dot{v}(t)|\}$. By the Schauder fixed point theorem, we conclude that $I$ has a fixed point $u_{*}$ in $F_{\varepsilon, T}$. By construction, $\xi+u_{*}$ is an orbit for the $n$ particle system with initial conditions $\xi\left(T_{j}\right)+u_{*}\left(T_{j}\right)=x\left(T_{j}\right), \quad \dot{\xi}\left(T_{j}\right)+\dot{u}_{*}\left(T_{j}\right)=\dot{x}\left(T_{j}\right)$. By uniqueness of solutions $\xi+u_{*}=x$, hence $x-\xi \in F_{\varepsilon, T}$. 
Let us fix a $T$ such that $x-\xi \in F_{\varepsilon, T}$ and such that

$$
\begin{aligned}
& x_{j}\left(t_{j}-T\right)-x_{j-1}\left(t_{j}-T\right)>R, \\
& \left(x_{j}\left(t_{j}-T\right)-x_{j-1}\left(t_{j}-T\right)\right)^{1+\alpha}>1+C_{2} / \varepsilon\left(b_{n-j+1}^{-}-b_{n}^{-}\right),
\end{aligned}
$$

where $C_{2}$ is the constant $C_{N}$ occurring in Lemma 1 with $N=2$. Define

$$
t^{\prime}=t_{j}-T \text {. }
$$

We define $X(t)$ to be the solution of (4.15) determined by the initial conditions

$$
X\left(t^{\prime}\right)=x\left(t^{\prime}\right), \quad \dot{X}\left(t^{\prime}\right)=\dot{x}\left(t^{\prime}\right) .
$$

Exactly analogously to the proof of Lemma 3 one shows that, as $a_{n}^{-} \rightarrow \infty$,

$$
\dot{X}(t)-\dot{x}(t)=O\left(\left(a_{n}^{-}\right)^{-1-\alpha}\right), \quad X(t)-x(t)=O\left(\left(a_{n}^{-}\right)^{-\alpha}\right) \quad \text { uniformly on } T_{j} \leqq t \leqq t^{\prime} .
$$

By the symmetry properties of the relative motion of the two particle system there exists a unique $t^{\prime \prime}>t^{\prime}$ such that

$$
\begin{aligned}
\dot{X}_{j-1}\left(t^{\prime \prime}\right) & =\dot{X}_{j}\left(t^{\prime}\right), \\
\dot{X}_{j}\left(t^{\prime \prime}\right) & =\dot{X}_{j-1}\left(t^{\prime}\right), \\
X_{j}\left(t^{\prime \prime}\right)-X_{j-1}\left(t^{\prime \prime}\right) & =X_{j}\left(t^{\prime}\right)-X_{j-1}\left(t^{\prime}\right) .
\end{aligned}
$$

Claim. $t^{\prime \prime}-t^{\prime}=O(1)$ as $a_{n}^{-} \rightarrow \infty$.

Proof. Let $\left(\alpha_{j-1} \alpha_{j}\right)$ and $\left(\beta_{j-1}, \beta_{j}\right)$ be the asymptotic phases and velocities of $\left(X_{j-1}(t), X_{j}(t)\right)$ as $t \rightarrow-\infty$. There exists a constant $c$ independent of $a_{n}^{-}$such that

$$
\begin{aligned}
& \left|\left(X_{j-1}(t), X_{j}(t)\right)-\left(\beta_{j-1}, \beta_{j}\right)\right| \leqq c\left|t-t^{\prime}\right|^{-1-\alpha}, \\
& \left|\left(X_{j-1}(t), X_{j}(t)\right)-\left(\alpha_{j-1}, \alpha_{j}\right)-\left(\beta_{j-1}, \beta_{j}\right) t\right| \leqq c\left|t-t^{\prime}\right|^{-\alpha} .
\end{aligned}
$$

Let $t_{j}^{\prime}=\frac{\alpha_{j}^{-}-\alpha_{j-1}^{-}}{\beta_{j-1}^{-}-\beta_{j}^{-}}$be the time where the asymptotic straight lines cross. From (4.15), (4.21), (4.23), and $t^{\prime}-T_{j}=O\left(a_{n}^{-}\right)$it follows that $t_{j}^{\prime}-t_{j}=O\left(\left(a_{n}^{-}\right)^{-\alpha}\right)$ as $a_{n}^{-} \rightarrow \infty$. By symmetry of the two particle relative motion, $t^{\prime}=t_{j}^{\prime}-T^{\prime}, t^{\prime \prime}=t_{j}^{\prime}+T^{\prime}$, where by (4.19) $T^{\prime}=t_{j}^{\prime}-t_{j}+T=O\left(\left(a_{n}^{-}\right)^{-x}\right)+T=O(1)$ as $a_{n}^{-} \rightarrow \infty$. Therefore $t^{\prime \prime}-t^{\prime}=2 T^{\prime}=O(1)$ as $a_{n}^{-} \rightarrow \infty$.

We only sketch the remaining part of the proof of Lemma 4 , since from now on one can proceed exactly analogous to the proof of Lemma 3.

Exactly analogous as in the proof of Lemma 3 one proves that

$$
\dot{X}-\dot{x}=O\left(\left(a_{n}^{-}\right)^{-2-\alpha}\right), \quad X-x=O\left(\left(a_{n}^{-}\right)^{-2-\alpha}\right) \quad \text { on }\left[t^{\prime}, t^{\prime \prime}\right] .
$$

The proof of the estimates $\dot{x}-\dot{X}=O\left(\left(a_{n}^{-}\right)^{-1-\alpha}\right)$ and $x-X=O\left(\left(a_{n}^{-}\right)^{-\alpha}\right)$ on $t^{\prime \prime} \leqq t \leqq T_{j-1}$, is analogous to the proof of Lemma 3. In order to apply the Gronwall inequality we first have to determine the rough behaviour of $x(t)$. To this end we define the space $F_{\varepsilon}$ and the operator $I$ by

$$
\begin{aligned}
& F_{\varepsilon}=\left\{u \in C^{1}\left(\left[t^{\prime \prime}, T_{j-1}\right], \mathbb{R}^{n)}\left|u\left(t^{\prime \prime}\right)=0, \dot{u}\left(t^{\prime \prime}\right)=0,\right| \dot{u}(t) \mid \leqq \varepsilon \quad \text { on } t^{\prime \prime} \leqq t \leqq T_{j-1}\right\},\right. \\
& (I u)(t)=-\int_{t^{\prime \prime}}^{t}(t-s) \operatorname{grad} V^{(n)}(\zeta(s)+u(s)) d s \quad\left(u \in F_{\varepsilon}\right),
\end{aligned}
$$


where $\zeta(s)=x\left(t^{\prime \prime}\right)+\left(s-t^{\prime \prime}\right) \cdot \dot{x}\left(t^{\prime \prime}\right)$.

Exactly as in the proof of Lemma 3 before it can be showed that $I$ possesses in $F_{\varepsilon}$ a fixed point $u_{*}$, which implies that $x-\zeta=u_{*} \in F_{\varepsilon}$. The knowledge about $x-\zeta \in F_{\varepsilon}$ allows us to apply the Gronwall inequality and to obtain as in the proof of Lemma 3 the desired estimates.

Proof of the theorem. From Lemmas 2 and 3 it follows that

$$
\begin{aligned}
& x\left(T_{1}\right)=a^{+}+b^{+} T_{1}+O\left(\left(T_{1}-a_{n}^{-} /\left(b_{n-1}^{-}-b_{n}^{-}\right)\right)^{-\alpha}\right)=A_{1}+B_{1} T_{1}+o(1), \\
& \dot{x}\left(T_{1}\right)=b^{+}+O\left(\left(T_{1}-a_{n}^{-} /\left(b_{n-1}^{-}-b_{n}^{-}\right)\right)^{-1-\alpha}\right)=B_{1}+O\left(\left(a_{n}^{-}\right)^{-1-\alpha}\right) \text { as } a_{n}^{-} \rightarrow \infty .
\end{aligned}
$$

This implies, since $T_{1}-a_{n}^{-} /\left(b_{n-1}^{-}-b_{n}^{-}\right)=\frac{1}{2} \cdot a_{n}^{-} /\left(b_{n-1}^{-}-b_{n}^{-}\right) \geqq O\left(a_{n}^{-}\right), B_{1}-b^{+}$ $=O\left(\left(a_{n}^{-}\right)^{-1-\alpha}\right)$, that $a^{+}=A_{1}+o(1)$ as $a_{n}^{-} \rightarrow \infty$.

The definition of $A_{1}$ implies that

$$
\begin{aligned}
a_{n-k+1}^{+}-a_{k}^{-}= & -\sum_{j<h} \delta\left(b_{j}^{-}-b_{k}^{-}\right) \\
& +\sum_{j>k} \delta\left(b_{k}^{-}-b_{j}^{-}\right)+o(1) \text { as } a_{n}^{-} \rightarrow \infty \text { for } k=1, \ldots, n .
\end{aligned}
$$

But the right-hand side of the above equation is independent of $a_{n}^{-}$as a consequence of the symplecticity of the scattering map, hence the $o$ (1) term in the above equation is identically zero. This proves Theorem 4.

Note Added. With the exception of Theorem 4 the results of this paper are now over five years old. Since the recent papers $[19,20]$ are partially based on our results, we decided to publish this paper as well.

Acknowledgement. This paper is an elaboration of my diploma thesis done with Prof. J. Moser, to whom I want to express my gratitude for suggesting the problem on the integrable systems and for many helpful discussions.

\section{References}

1. Airault, H., McKean, H.P., Moser, J.: Rational and elliptic solutions of the Korteweg-de Vries equation and a related many-body problem. Commun. Pure Appl. Math 30, 95-148 (1977)

2. Calogero, F., Marchioro, C., Ragnisco, O.: Exact solution of the classical and quantal onedimensional many-body problems with the two-body potential $V_{a}(x)=g^{2} a^{2} / \sinh ^{2}(a x)$. Lett. Nuovo Cimento 13, 383-387 (1975)

3. Choodnovsky, D.V., Choodnovsky, G.V.: Nuovo Cimento 40B, 39 (1977)

4. Galperin, G.A.: Elastic collisions of particles on a line. Uspekhi Mat. Nauk 33:1, 211-212 (1978), (Russian); Russ. Math. Surv. 33:1, 199-200 (1978), (English transl.)

5. Galperin, G. A.: On systems of locally interacting and repelling particles moving in space. Trudy MMO 43, 142-196 (1981), (Russian); Trans. Moscow Math. Soc., Issue 1, 159-214 (1981) (English transl.) 
6. Galperin, G.A.: Asymptotic behaviour of particle motion under repulsive forces. Commun. Math. Phys. 84, 547-556 (1982)

7. Gardner, C.S., Greene, J.M., Kı uskal, M.D., Miura, R. M.: Korteweg-de Vries equation and generalizations. VI. methods for exact solution. Commun. Pure Appl. Math. 27, 97-133 (1974)

8. Herbst, I.W.: Classical scattering with long range forces. Commun. Math. Phys. 35, 193-214 (1974)

9. Hunziker, W.: The $S$-matrix in classical mechanics. Commun. Math. Phys. 8, 282-299 (1968)

10. Hunziker, W.: Scattering in classical mechanics, Scattering theory in Mathematical Physics. J.A. La Vita, J.-P. Marchand (eds.) pp. 79-96, 1974

11. Khimchenko, N.G., Sinai, Ya.G.: On the description of classical reflectionless potentials. Rep. Math. Phys. 20, 53-63 (1984)

12. Kulish, P.P.: Factorization of the classical and the quantum $S$ matrix and conservation laws. Theor. Math. Phys. 26, 132-137 (1976)

13. Moser, J.: Finitely many mass points on the line under the influence of an exponential potential - an integrable system. Lecture Notes in Physics, vol. 38, pp. 467-497. Berlin, Heidelberg, New York: Springer 1975

14. Moser, J.: Three integrable Hamiltonian systems connected with isospectral Deformations. Adv. Math. 16, 197-220 (1975)

15. Moser, J.: The scattering problem for some particle systems on the line. Lecture Notes in Mathematics, vol. 597, pp. 441-463. Berlin, Heidelberg, New York: Springer 1977

16. Simon, B.: Wave operators for classicle particle scattering. Commun. Math. Phys. 23, 37-48 (1971)

17. Sinai, Ya.G.: Billiard trajectories in a polyhedral angle. Uspekhi Mat. Nauk 33:1, 229-230 (1978), (Russian), Russ. Math. Surv. 33:1, 219-220 (1978). (English transl.)

18. Vaserstein, L.N.: On systems of particles with finite-range and/or repulsive interactions. Commun. Math. Phys. 69, 31-56 (1979)

19. Gutkin, E.: Regularity of the scattering trajectories in classical mechanics. Commun. Math. Phys. 119, 1-12 (1988)

20. Gutkin, E.: Regularity of asymptotic velocities in classical mechanics, preprint

Communicated by J.-P. Eckmann

Received June 10, 1988; in revised form December 1, 1988 
